\title{
REVISIÓN DEL DIAGNÓSTICO DE TRATAMIENTO DE AGUAS DE PRODUCCIÓN EN CAMPOS PETROLEROS COLOMBIANOS
}

\section{Review of the diagnosis of production water treatment in Colombian petroleum fields}

\author{
Angie Tatiana Ortega Ramírez ${ }^{1}$, Yurleni Fernanda Arcila², Laura Marcela Vargas Díaz ${ }^{3}$ \\ ${ }^{1}$ Fundación Universidad de América. Docente Facultad de Ingenierías, Colombia. \\ ${ }^{2-3}$ Fundación Universidad de América. Estudiante Facultad de Ingenierías. Ingeniería Química, Colombia. \\ Email: ${ }^{1}$ angie.ortega@profesores.uamerica.edu.co, ${ }^{2}$ yurleni.arcila@estudiantes.uamerica.edu.co, \\ 3laura.vargas5@estudiantes.uamerica.edu.co
}

(Recibido Febrero 19 de 2020 y aceptado Junio 21 de 2020)

\section{Resumen}

El presente artículo plantea estudiar los métodos de gestión del recurso hídrico en la industria del petróleo. Para la investigación se revisa información científica obtenida en bases de datos como Scopus, Science Direct o Redalyc, e indagaciones extraídas de reportes gubernamentales, la legislación colombiana y de operaciones de empresas pertenecientes al sector de los hidrocarburos como Ecopetrol o Pacific, entre otras. Con los datos se clasifican los métodos convencionales y no convencionales de separación entre agua y petróleo crudo. Finalmente, se subrayan tres usos dados al agua de producción en Colombia: inyección, vertimiento superficial y adecuación como agua de riego en actividades agrícolas.

Palabras clave: agua subterránea, tratamiento, campo petrolero, petróleo.

\begin{abstract}
This article aims to study the methods of water resource management in the petroleum industry. This research looks for scientific information obtained in databases such as Scopus, Science Direct, or Redalyc, and inquiries extracted from government reports, Colombian legislation, and operations of companies belonging to the hydrocarbon sector such as Ecopetrol or Pacific, among others, are reviewed. With the data, conventional and non-conventional methods of separation between water and crude petroleum are classified. Finally, three uses given to the produced water in Colombia are highlighted: injection, surface discharge, and adaptation as irrigation water in agricultural activities.
\end{abstract}

Key words: groundwater, treatment, oil field, petroleum.

\section{INTRODUCCIÓN}

El agua de producción, también denominada de formación, alude a aquella obtenida junto con el petróleo y el gas. Se origina como una capa de agua natural localizada debajo de los hidrocarburos en los yacimientos de petróleo y gas [1]. Generalmente, esta contiene aceites y compuestos orgánicos solubles e insolubles, sólidos suspendidos y disueltos, metales pesados y varios químicos utilizados para el proceso de producción de petróleo [2]. Actualmente, esta industria origina una alta cantidad de agua en la fase de extracción de hidrocarburos, aproximadamente, más de 300 millones de barriles de agua se reportan diariamente en el mundo [3]. En promedio se generan de tres a cinco barriles de agua por cada barril de petróleo; sin embargo, en pozos considerados maduros esta cifra puede aumentar hasta una relación de entre diez y catorce barriles de agua [4]. En los campos petroleros se utiliza más del $60 \%$ del agua obtenida a diario en el mundo. [5]. Adicionalmente, se debe 
tener en cuenta que no solo el caudal de agua producida, también su composición, varía, dependiendo del pozo. En Colombia, particularmente, por cada barril de petróleo se producen, en promedio, trece barriles de agua [6]. En 2019 el volumen total de efluentes generado por las operaciones de Ecopetrol fue de 429.14 millones de metros cúbicos, los cuales incluyen: agua de producción (92.8\%) y agua residual industrial (7.2\%) [7]. Según BNamericas [8], los dos campos con mayor producción de petróleo en Colombia son Rubiales, en primer lugar, seguido por Castilla; ambos operados por la empresa Ecopetrol S. A. Tales campos generan altas cantidades de agua de producción.

Para el campo Rubiales se estimó una producción de 3 000000 BWPD (barriles de agua por día) en el año 2019 [7], y para el campo Castilla el corte de agua en marzo del 2019 fue de 1040000 BWPD [9].

Actualmente, las principales alternativas para la gestión del agua producida son:

\section{Inyección en Formaciones}

El agua producida se inyecta nuevamente en su propia formación o en otras. Esta opción a menudo requiere transporte de agua y tratamiento para reducir el ensuciamiento y el crecimiento bacteriano [10]. Sin embargo, este tipo de inyección puede darse manteniendo o aumentando la presión en el yacimiento o reinyectando a la formación no productora o sin efecto de recobro [7].

\section{Descarga al Medio Ambiente}

En este caso el agua producida puede descargarse en acuíferos siempre y cuando cumpla con las regulaciones de descarga en tierra y en altamar [10].

Reutilización en las Operaciones de la Industria Petrolera: En esta disposición el agua producida con un tratamiento mínimo se puede usar para operaciones de perforación y preparación en la industria petrolera [10], por ejemplo: Preparación de lodos de perforación, mantenimiento de pozos, sistemas contra incendios, refrigeración de equipos, generación de energía [7], entre otras.
Preparación de lodos de perforación, mantenimiento de pozos, sistemas contra incendios, refrigeración de equipos, generación de energía [7], entre otras.

\section{Aplicación en Usos Beneficiosos}

El agua producida puede ser dispuesta para uso en comunidades y sus actividades como el riego y cuidado del hábitat, tratamiento de agua industrial e incluso manejo de agua potable. Aunque, los usos beneficiosos de esta agua pueden implicar un tratamiento adicional más robusto con un costo elevado [10].

El agua producida tiene una composición compleja debida a la variedad de componentes presentes en ella; estos pueden clasificarse ampliamente en compuestos orgánicos e inorgánicos [11] incluidos aceites disueltos y dispersos, grasas, metales pesados, radionucleidos, productos químicos de tratamiento, sólidos de formación, sales, gases disueltos, productos a escala, ceras, microorganismos y oxígeno disuelto [10].

El carbono orgánico total (TOC) es un indicador de la cantidad total de materia orgánica presente en el sedimento y se utiliza como indicador de la riqueza de la fuente con respecto a la cantidad de hidrocarburos que puede generar ese sedimento [12]. En el agua producida varía de menos de 0.1 a más de $11000 \mathrm{mg} / \mathrm{L}$, gran parte de estos consiste en una mezcla de ácidos carboxílicos de bajo peso molecular, como los ácidos fórmicos, acético, propanoico, butanoico, pentanoico y hexanoico [13].

En los sólidos disueltos (componentes inorgánicos) predominan cationes de sodio $\left(\mathrm{Na}^{+}\right)$y aniones de cloruro $(\mathrm{Cl})$, razón por la cual la salinidad del agua de producción es similar a la salinidad del agua de mar [13]. No obstante, también se puede encontrar otro tipo de cationes como el calcio $\left(\mathrm{Ca}^{+}\right)$, magnesio $\left(\mathrm{Mg}^{+}\right)$, hierro $\left(\mathrm{Fe}^{+}\right) \mathrm{y}$, en una menor proporción, bario $\left(\mathrm{Ba}^{2+}\right)$, potasio $\left(\mathrm{K}^{+}\right)$, estroncio $\left(\mathrm{Sr}^{+}\right)$, aluminio $\left(\mathrm{Al}^{3+}\right)$ y litio $\left(\mathrm{Li}^{+}\right)$. Otros aniones presentes son: bicarbonato $\left(\mathrm{HCO}_{3}\right)$, carbonato $\left(\mathrm{CO}_{3}\right)$ y sulfatos $\left(\mathrm{SO}_{4}\right)[14]$.

Entre los aceites y dispersos se encuentran los hidrocar- 
buros derivados del petróleo, que consisten en estructuras compuestas de carbono e hidrógeno.

Se trata de los compuestos de mayor preocupación ambiental en el agua producida. Se clasifican en hidrocarburos saturados y aromáticos. Los aromáticos de un anillo son los más abundantes, en ellos se pueden encontrar benceno, tolueno, etilbenceno y xilenos (BTEX). Los aromáticos policíclicos (HAP) son los hidrocarburos de petróleo de mayor preocupación ambiental en el agua producida debido a su toxicidad y persistencia en el medio marino.

Otros compuestos que se pueden encontrar en el agua de producción son los metales pesados, suelen estar en concentraciones sustancialmente más altas que las presentadas en el agua de mar. Los metales encontrados con mayor frecuencia incluyen bario, hierro, manganeso, mercurio y zinc [13].

Los productos químicos de producción pueden ser compuestos puros o compuestos que contienen ingredientes activos disueltos en un solvente o codisolvente y se utilizan para inhibir la corrosión, la formación de hidratos, posición de incrustaciones, la producción de espuma y el crecimiento bacteriano. El destino de estos químicos es difícil de determinar porque algunos ingredientes activos se consumen durante el proceso [10].

Los principales gases disueltos en el agua producida son el dióxido de carbono $\left(\mathrm{CO}_{2}\right)$, el oxígeno $\left(\mathrm{O}_{2}\right)$ y el sulfuro de hidrógeno $\left(\mathrm{H}_{2} \mathrm{~S}\right)$, los cuales se forman naturalmente por las actividades bacterianas o por reacciones químicas en el agua [10].

La magnitud de la carga contaminante del agua residual de producción de hidrocarburos se ve reflejada también en parámetros como demanda biológica de oxígeno (DBO), demanda química de oxígeno (DQO), sólidos suspendidos totales, nitrógeno y fósforo total [15].

La importancia del tratamiento del agua de producción reside en el impacto ambiental que genera en cuerpos de agua y poblaciones circundantes, cuando se realizan vertimientos superficiales [16], esto debido a que los hidrocarburos de petróleo flotantes en la superficie reducen la transferencia de oxígeno atmosférico al agua, lo que lleva a modificaciones específicas, y finalmente altera el equilibrio biológico de los sitios contaminados [17].

En cuanto al impacto en la salud humana, la exposición a compuestos BTEX puede ocurrir al beber agua contaminada (ingestión) y sus consecuencias se asocian con irritación de la piel, problemas del sistema nervioso central -SNC y efectos en el sistema respiratorio [18]. Además de esto, los metales pesados son extremadamente tóxicos para los seres humanos aún en cantidades pequeñas y son absorbidos por los crustáceos, asimismo, los sulfuros pueden matar a los peces, al igual que los aceites y grasas presentes en el agua [19].

Se debe tener en cuenta que no solo los vertimientos superficiales traen consigo afectaciones ambientales y en la salud humana, sino también los procesos de inyección de agua de producción en formaciones que, a largo plazo, pueden llegar a contaminar las aguas subterráneas [10].

De acuerdo con la información presentada anteriormente, el presente artículo parte de una revisión bibliográfica que busca establecer el diagnóstico del proceso de tratamiento de agua de producción en campos petroleros colombianos.

\section{METODOLOGÍA}

El presente artículo se realiza bajo una exhaustiva revisión de información de diversas bases de datos de artículos científicos como Scopus, Google Scholar, Redalyc y Science Direct, así como de informes de operación de empresas del sector de los hidrocarburos tales como Ecopetrol, Pacific y se vale de reportes gubernamentales, de la legislación colombiana, entre otros. Mediante la clasificación temática y de autores se cumplió el número de citas necesario para desarrollar el tema pertinente. 


\section{DESARROLLO}

Como se mencionó anteriormente, los campos con mayor índice de producción de agua en Colombia son Rubiales Rubiales y Castilla.

\subsection{Campo Rubiales}

Se encuentra ubicado en Colombia, en el sureste de la cuenca de los Ilanos Orientales, en el departamento del Meta, dentro del municipio de Puerto Gaitán, 365 km al este de la ciudad de Villavicencio y $482 \mathrm{~km}$ al sureste de Bogotá [20].

En 2019 este campo generó aproximadamente 3000000 de BWPD (Barrels of water per day) [7]. Debido al alto volumen de agua producida es necesario el tratamiento de estos efluentes para su correcta disposición final.

Este proceso inicia con una separación primaria, donde el crudo proveniente del manifold externo ingresa a los tanques de surgencia, que son separadores utilizados, principalmente, para retirar agua libre que pueda causar problemas como corrosión y formación de hidratos o emulsiones compactas [21]. El crudo separado con un menor corte de agua es dirigido a los tanques de cabeza, donde se separa el agua restante presente en el crudo. Posteriormente, es enviada junto con el agua proveniente de los fondos de los tanques de surgencia hacia los skim tanks para el proceso de desnatado (skimming). Estos equipos están normalmente diseñados para proveer tiempos de residencia largos durante los cuales la coalescencia y la separación por gravitación pueden ocurrir, de esta forma se logra retirar el aceite de la superficie del agua sin alterar sus características físicas o químicas [22]. Los tanques desnatadores pueden ser usados como tanques atmosféricos, recipientes a presión y tanques de aumento sobre otros equipos de tratamiento de agua de producción [23]. Después de este proceso el agua es transportada de forma continua por medio de bombas hacia los trenes de tratamiento, conformados por una celda de flotación de natas, filtros de tipo cáscara nuez y decantadores verticales. Las celdas de flotación estándar pueden utilizar dos técnicas diferentes (DAF - IAF) para retirar altos porcentajes de aceite, grasas y sólidos suspendidos del agua de producción [24]. En este proceso se inyecta aire en el agua producida.

Las partículas suspendidas y las gotas de aceite se unen a las burbujas de aire. Así, a medida que se elevan se forma espuma en la superficie del agua que se elimina mediante desnatado, mientras que el agua clarificada es recogida en el fondo de la zona de flotación [10]. Continúa hacia los filtros de cáscara nuez, atravesando un medio poroso filtrante donde se separan el petróleo disperso en agua, los sólidos suspendidos, y las grasas y aceites [25].

El petróleo se adhiere a las pequeñas partículas de cáscara nuez, permitiendo que el agua fluya hacia el interior del filtro y salga del equipo [26]. Se debe tener en cuenta que la capacidad de los filtros es limitada y se controla mediante el diferencial de presión que genera el medio poroso [25]. Por esta razón, se realiza un ciclo de retrolavado, consistente en un proceso de limpieza, invirtiendo el flujo a través de la unidad para remover y desplazar las trazas de crudo acumuladas. Esta acción inversa expande el medio filtrante, limpiándolo por acción hidráulica y fricción entre partículas [27], a la salida del proceso de retrolavado el agua es dirigida a los decantadores gravitatorios; estos equipos se utilizan para la separación de líquidos no miscibles (natas, agua clarificada y lodos) de densidades diferentes [28]. La mezcla permanece inmóvil de cuatro a seis horas con el fin de lograr la separación gravitacional. El agua obtenida en el proceso de decantación se denomina agua clarificada; ingresa luego a una piscina de agua de retrolavado donde se realiza una pulimentación para la separación de lodos y natas aún presentes en el agua de producción. Finalmente, el agua proveniente de las piscinas de retrolavado es dirigida a las piscinas de agua tratada, donde, de igual forma, ingresa directamente el agua filtrada obtenida después del proceso de filtro cáscara nuez, y se realiza su disposición final de acuerdo a la necesidad.

Actualmente, el campo Rubiales cuenta con dos alternati 
vas de gestión para el agua tratada. La primera es el vertimiento superficial, y la segunda, la inyección sin efecto de recobro o a formación no productora.

\subsection{Campo Castilla}

El campo Castilla se encuentra localizado en el sector suroccidental de la cuenca de los llanos Orientales a una distancia aproximada de $54 \mathrm{~km}$ al sur de la ciudad de Villavicencio, departamento del Meta, en las jurisdicciones municipales de Castilla la Nueva, Acacias Guamal y San Martín; y dentro de la cuenca de los ríos Guamal y Orotoy [29].

Durante marzo de 2019 el corte de producción de agua diario fue de aproximadamente 1040000 BWPD [9]. En el caso específico de la estación Castilla II el agua de producción proveniente de los tanques de surgencia y tanques de lavado ingresa a las celdas de placas corrugadas (CPI), siendo esta la primera etapa en el STAP (Sistema de Tratamiento de Aguas de producción Petroleras) de tratamiento del agua, donde se efectúa una separación de tres fases (agua, lodos y aceites) por diferencia de densidades [30]. Como estos equipos no tienen partes móviles presentan una mayor área de distribución para el efluente de agua residual [31].

Este efluente obtenido es enviado a las celdas de flotación de gas inducido (IGF), donde se utiliza una técnica de separación gravitacional acelerada, en la que las burbujas de gas son inducidas a una corriente de fase acuosa, ya sea por el uso de un dispositivo eductor o por un vórtice creado por un rotor mecánico (impulsor) [32]. En dichas celdas se separan y recuperan el aceite y las partículas sólidas presentes en el agua de producción a través de la creación de burbujas de gas finas (diámetro inferior a 50 micras), que se dispersan. Las partículas se adhieren a las burbujas de gas y flotan en la superficie, formando una capa de espuma que se remueve mediante los desnatadores de la celda [33].

El agua clarificada continúa hacia los filtros de cáscara nuez donde se elimina el aceite remanente, lodos e impurezas, después es enviada a las torres de enfriamiento donde, mediante boquillas de aspersión, se atomiza y desciende debido a la acción gravitacional. Gracias al fenómeno de transferencia de calor y masa [34], al entrar en contacto con el aire, se posibilita la disminución de su temperatura para, continuamente y por un canal de entrega, conducirla hacia las piscinas de estabilización y aspersión, las cuales representan la última etapa en el proceso de tratamiento del agua, y allí se busca, por medio de la aspersión, disminuir la temperatura, oxigenar el agua y reducir la cantidad de fenoles disueltos [30]. El agua tratada puede disponerse para diferentes fines según las necesidades de la empresa, entre los que se cuentan vertimiento superficial, inyección con efecto de recobro, inyección sin efecto de recobro, reutilización en operaciones y reúso.

Debido a la problemática generada por el déficit de recursos hídricos, el reúso del agua ha sido considerado una alternativa de gestión para contrarrestar este hecho [35]. Si bien el agua es un recurso renovable, en ciertas zonas la demanda agrícola, el crecimiento poblacional y los cambios climáticos (períodos de sequía) han causado que el consumo de agua dulce exceda su capacidad de reposición natural [36].

La utilización del agua en la agricultura representa como mínimo dos tercios de su consumo global [36], el uso de agua regenerada en agricultura permite aliviar la presión ejercida sobre los sistemas de abastecimiento de agua dulce y conservarlos para un fin de mayor valor económico y social y, al mismo tiempo, los agricultores reciben un suministro de agua fiable y rico en nutrientes [35]. El reciclaje del agua puede ofrecer un "triple dividendo" para los usuarios urbanos, agricultores y el medio. La disponibilidad sustancial de agua producida, sumada con la necesidad de contar con alternativas de eliminación más económicas conduce a los investigadores a estudiar la reutilización del agua producida para irrigación, uso industrial y otras aplicaciones [36].

En 2019 mediante un estudio científico realizado por la Corporación Colombiana de Investigación Agropecuaria (Corpoica, hoy Agrosavia) se concluyó que el agua de 
producción tratada por Ecopetrol en sus campos Castilla y Apiay es apta para el uso en actividades agrícolas, pecuarias y en el riego de cultivos de porte forestal. Se reusaron 1.2 millones de metros cúbicos de agua de producción tratada en el campo Castilla para el riego del Área de Sostenibilidad Agroenergética (ASA) [7].

Debido a que el proceso de irrigación requiere elevados volúmenes de agua que cuenten con los parámetros de calidad establecidos según las normativas de cada país es necesario el desarrollo de sistemas de tratamiento robustos que permitan obtener un agua que cumpla con los criterios específicos para asegurar la calidad apropiada con el fin de ser dispuesta en riego, algunos de los más importantes son: la salinidad, relación de adsorción de sodio (SAR) y la conductividad eléctrica [12]. Con esto se describen algunos de los tratamientos utilizados actualmente y las nuevas tecnologías enfocadas a la obtención de un agua que cumpla con los parámetros de calidad para su disposición final en riego.

\subsection{Tratamientos Físicos}

Estos tratamientos son aquellos en los que no se generan sustancias nuevas, sino que se concentran los contaminantes al evaporar el agua o filtrar sólidos de un tamaño considerable [37]. A continuación, se describen los procesos de tratamiento físico utilizados actualmente en la industria.

\subsubsection{Adsorción. Es un proceso de transferencia de masa,} mediante el cual las sustancias presentes en los fluidos son transferidas a un medio sólido [38]. Esta es una de las operaciones unitarias más eficientes para alcanzar una mejor calidad del agua, debido a que logra reducir la concentración de los contaminantes del agua producida a niveles muy bajos [39]. La eliminación de compuestos como el manganeso, hierro, TOC, BTEX, petróleo y de hasta el $80 \%$ de los metales pesados se puede lograr mediante el proceso de adsorción [12].

Sin embargo, una de las principales desventajas de este método es el costo de instalación y mantenimiento del sistema, pero este inconveniente puede resolverse por medio del uso de adsorbentes más económicos tales como la cáscara nuez y el carbón activado, que pueden hacer que el proceso sea más competitivo [39]. Los filtros de cáscara de nuez funcionan con los ciclos de proceso de filtración y retrolavado [40].

Otra desventaja del proceso de adsorción es el requerimiento de un depósito de residuos para su eliminación y la de los medios gastados a través del proceso de regeneración [39].

\subsubsection{Ciclones. Los hidrociclones se utilizan para separar} compuestos como arena y aceite, contenidos en la corriente de agua de producción y se basan en las diferencias de densidades. El agua de producción contiene una alta cantidad de sólidos suspendidos y gotas pequeñas de aceite, como también, tensoactivos. Dependiendo del modelo empleado para el hidrociclón, este puede remover partículas con un diámetro entre 5 y $15 \mu \mathrm{m}$; sin embargo, no pueden removerse componentes solubles en el agua [41]. Algunas de las ventajas de este tipo de equipos son su larga vida útil y el innecesario uso de productos químicos o pretratamiento del agua de alimentación. Por otro lado, una desventaja importante de esta tecnología es la generación de grandes suspensiones de residuos sólidos concentrados [10] y su baja eficiencia, además, su incapacidad de remover compuestos disueltos [42].

3.3.3. Evaporación. La evaporación se propone como un tratamiento para la recuperación o separación de materiales valiosos o peligrosos (metales pesados) [43], especialmente, para aguas residuales salinas con contenido de aceites [12]. Su robustez permite evitar otro procedimiento físico o químico posterior, ya que mediante la adición de calor latente al agua de alimentación se genera vapor que se condensa en agua pura [15].

3.3.4. Flotación mejorada. La flotación por aire disuelto (DAF) es un proceso conocido en el tratamiento de aguas debido a su capacidad para la eliminación eficiente de una serie de contaminantes como partículas finas, 
ultrafinas coloidales, precipitados orgánicos e inorgánicos, iones, microorganismos, proteínas, emulsiones de aceite en agua y aceite disperso [44]. El aire se disuelve a alta presión en un saturador, y las microburbujas se forman cuando se libera agua en la celda de flotación a presión atmosférica. Las microburbujas se adhieren a las partículas presentes en el agua de producción, permitiendo así aumentar su flotabilidad, al llevarlas hacia la superficie [45].

Algunas de las ventajas de este proceso son su habilidad para tratar altos volúmenes de efluentes (100-600 m3 h-1), pequeña área ocupada, entrega una excelente calidad de agua, genera lodos más espesos y tiene una rápida puesta en marcha; sin embargo, una de las desventajas es el alto consumo energético y los altos costos de operación comparados con otras técnicas de tratamiento físicas [44].

3.3.5. Electrodiálisis. La electrodiálisis (ED) es una técnica de separación impulsada por cargas electroquímicas donde las sales disueltas en el agua de producción son cationes y aniones que pueden unirse a electrodos específicos con una carga opuesta [46]. En esta tecnología las membranas de intercambio iónico se organizan alternativamente en un campo de corriente continua [47].

Este método es adecuado para la recuperación de agua producida con bajas concentraciones de SDT (Sólidos totales disueltos), pero es poco probable que sea rentable para el tratamiento de aguas producidas concentradas [5], además no es tan económicamente competitivo como otras separaciones por membrana, debido al alto costo de los electrodos y las membranas, así como a una vida útil relativamente corta [47].

\subsection{Tratamientos Químicos}

En los tratamientos de tipo químico se generan nuevas sustancias mediante reacciones químicas que permiten la disminución de compuestos contaminantes presentes en el agua de producción [37]. A continuación, se presenta una breve descripción de los tratamientos químicos implementados actualmente en la industria y algunas nuevas tecnologías.

3.4.1. Precipitación química. Se usa para eliminar componentes iónicos del agua mediante la adición de contraiones para reducir la solubilidad [48], además logra eliminar metales disueltos en el agua de producción. La eficacia del proceso depende de varios factores, entre ellos, la concentración de metales iónicos presentes en la solución, el precipitante utilizado y la presencia de otros constituyentes que pueden inhibir la reacción de precipitación [49].

3.4.2. Procesos electroquímicos. Los reactores electroquímicos son aparatos de transformación de materiales forzados por la corriente eléctrica. La oxidación ocurre en el ánodo y la reducción en el cátodo [50]. Este proceso está basado en la mejora de las reacciones químicas que implican la generación o el uso de electricidad. La electroquímica es una tecnología verde, relativamente, económica en comparación con otros tratamientos actuales aplicados al agua de producción. No genera desechos secundarios ni requiere el uso de productos químicos adicionales y ofrece un agua de producción tratada de mejor calidad para usos beneficiosos [12]. Entre los procesos electroquímicos más conocidos están la electrodeposición, electrocoagulación, electroflotación y electrooxidación [51].

3.4.3. Demulsificantes. Existen varios métodos para la demulsificación del petróleo crudo, entre ellos, electrosedimentación, demulsificación supersónica, centrifugación y demulsificación química con demulsificantes [52]. La demulsificación química es el método más utilizado para tratar emulsiones de aceite en agua e implica el uso de aditivos químicos para acelerar el proceso de ruptura de la emulsión [53]. Los tensoactivos inducen a la estabilización de las emulsiones aceite-agua, al reducir la tensión interfacial entre estas y el potencial zeta en las superficies de las gotas de aceite [12]. Los demulsificantes comerciales son tensoactivos poliméricos como copolímeros de poli (óxido de etileno) (PEO) y poli (óxido de propileno) (PPO), además de alquilfenol -resinas de formaldehído o mezclas de diferentes sustancias tensoactivas [52]. En 
la mayoría de los aceites crudos, los sólidos tales como los sulfuros de hierro, limos, arcilla, lodo de perforación, parafina, etcétera complican el proceso de demulsificación [5].

\subsubsection{Intercambio iónico. Las membranas de intercambio} iónico son una clase importante de membranas poliméricas densas que soportan cargas fijas en la matriz del polímero. Estas membranas permiten el paso selectivo de iones con carga opuesta (contraiones), mientras obstruyen los iones cargados de manera similar (coiones). Este proceso se ha establecido como un componente clave en la desalinización y electrólisis del agua [54].

\subsubsection{Oxidación química. La oxidación química es una} tecnología conocida y útil para el tratamiento de efluentes con compuestos refractarios [55], este proceso ocurre mediante la transferencia de electrones de un reactivo oxidante a una especie química que se oxida [56]. Actualmente, se desarrollan tratamientos de aguas de producción con el fin de reducir la cantidad de hidrocarburos, además de eliminar el color, olor, DQO y DBO presentes en el agua de producción [12]. Las técnicas de separación convencionales no eliminan los compuestos orgánicos disueltos, por lo tanto, deben implementarse varias oxidaciones, entre ellas biológicas y químicas [57].

Con peróxido de hidrógeno. El peróxido de hidrógeno $(\mathrm{H} 2 \mathrm{O} 2)$ es un oxidante fuerte que puede reaccionar directamente con los compuestos orgánicos presentes en el agua de producción. Resulta aún más efectivo cuando se usa en combinación con otra energía o reactivos (oxidación avanzada), capaces de disociar el peróxido en un radical hidroxilo que actuará como agente oxidante [12].

Con ozono. El ozono es un oxidante y viricida muy fuerte [58]. Es uno de los oxidantes más eficaces, utilizado durante mucho tiempo para el tratamiento de agua potable para la desinfección, el tratamiento de olores y la eliminación del color causado por sustancias húmicas, y para de la degradación de varios productos químicos orgánicos incluidos los PAHs (Hidrocarburo aromático policiclico)[59].
La ozonización implica un proceso escalonado mediante el cual se introducen progresivamente átomos de oxígeno en el compuesto, que generan moléculas más pequeñas, las cuales contienen un mayor porcentaje de oxígeno en su estructura química en forma de grupos funcionales hidroxilo, carboxilo o aldehído, por lo que resultan más susceptibles a la biodegradación [60].

3.4.6. Oxidación avanzada. Durante este proceso los radicales hidroxilos $\left(\mathrm{OH}^{-1}\right.$ se generan en cantidades suficientes [61] que permiten, gracias a su reactividad y alta selectividad, transformar diversos contaminantes orgánicos e inorgánicos, inhibiendo así la posterior generación de residuos tóxicos [15].

La mayoría de estos procesos, excepto la ozonización simple $\left(\mathrm{O}_{3}\right)$, utiliza una combinación de oxidantes fuertes, radiación ultravioleta (UV), ultrasonido (US) o haz de electrones y catalizadores, etcétera. Algunos de los sistemas de oxidación avanzada típicos son: $\mathrm{O}_{3} / \mathrm{UV}, \mathrm{H}_{2} \mathrm{O}_{2} / \mathrm{UV}$, rayo de electrones, ultrasonido (US), $\mathrm{H}_{2} \mathrm{O}_{2} / \mathrm{US}, \mathrm{UV} / \mathrm{US}, \mathrm{H}_{2} \mathrm{O}_{2} /$ $\mathrm{Fe}^{2+} / \mathrm{UV}$ (fotofenton), $\mathrm{O}_{3} / \mathrm{H}_{2} \mathrm{O}_{2}, \mathrm{O}_{3} / \mathrm{OH}, \mathrm{H}_{2} \mathrm{O}_{2} / \mathrm{Fe}^{2+}$ (Fenton), entre otros [55].

\subsection{Tratamientos Biológicos}

Los tratamientos biológicos son extremadamente efectivos en varios tipos de moléculas disueltas e incluyen los hidrocarburos de petróleo, y, por lo tanto, son muy útiles para reducir los niveles de contaminación orgánica en el agua producida [62]. En la oxidación biológica, los compuestos orgánicos disueltos y el amoniaco son convertidos en agua, $\mathrm{CO} 2$ y nitratos/nitritos, respectivamente, por microorganismos como bacterias, algas, hongos y protozoos, mediante mecanismos de biodegradación y biofloculación [12]. Entre los procesos y tecnologías biológicas para el tratamiento de agua de producción se encuentran los reactores de secuenciación por lotes, los filtros biológicos aireados, los lodos activados y humedales artificiales. Los procesos biológicos suelen ser más eficaces para un agua de alimentación con un DQO $<400 \mathrm{mg} / \mathrm{L}$, un $\mathrm{DBO}<50 \mathrm{mg} / \mathrm{L}$, nivel de aceite $<60 \mathrm{mg} / \mathrm{L}$ y una concentración de cloruro $<2600 \mathrm{mg} / \mathrm{L}$ del agua producida [39]. 
Cabe resaltar que los procesos de tratamiento biológico son considerados unos de los más económicos para la remoción de contaminantes presentes en el agua [39].

\subsection{Tratamientos de membrana}

Las membranas son películas microporosas con índices de poros específicos, que separan selectivamente un fluido de sus componentes [10]. La separación con membranas se produce debido a la existencia de un gradiente a través de estas. Los procesos de separación por membrana pueden dividirse en las impulsadas por presión y las no impulsadas por presión [63]. El primer proceso se basa en el tamaño del poro de la membrana [5], en donde se genera un gradiente de presión, denominado presión transmembrana [63], esto con el fin de separar los contaminantes presentes en la corriente de alimentación. Los procesos de separación por membrana impulsados por presión incluyen microfiltración (MF), ultrafiltración (UF), ósmosis inversa (RO) y nanofiltración (NF). La MF se enfoca en la separación de partículas suspendidas; la UF, en la separación de macromoléculas, y la RO, en la separación de componentes iónicos y disueltos [5]. A continuación, se hace una descripción detallada de cada de las tecnologías mencionadas anteriormente.

3.6.1. Microfiltración (MF). La separación por microfiltración tiene un tamaño de poro más grande $(0.1-3 \mu \mathrm{m})$ y se usa generalmente para la eliminación de sólidos suspendidos y la reducción de la turbidez [10], a presiones entre los 0.5 y 3 bar.

Esta tecnología se implementa como etapa de limpieza, de concentración o como pretratamiento para la nanofiltración o a la ósmosis inversa [64].

3.6.2. Ultrafiltración (UF). La UF es el método más eficaz para la eliminación de hidrocarburos del agua producida en comparación con los métodos de separación convencionales, gracias a su alta eficiencia de remoción de aceite, bajos costos de energía, pequeños requerimientos de espacio y la inexistencia de aditivos químicos [5]. Esta tecnología es más eficaz que la MF para la eliminación de hidrocarburos, sólidos en suspensión y compuestos disueltos en el agua de producción [10]. Las membranas de UF permiten la eliminación de partículas coloidales más pequeñas, virus y macromoléculas como proteínas y algunos ácidos húmicos [63]. Tiene un tamaño de poro entre 0.01 y $0.1 \mu \mathrm{m}$ y trabajan a presiones inferiores que los 1000kPa [64].

Tanto la MF como la UF operan a baja presión transmembrana y pueden servir como pretratamiento para la desalinización, pero no pueden eliminar la sal del agua [10].

3.6.3. Nanofiltración (NF). La NF es un proceso de membrana impulsado por presión, implica presiones entre 5 y 20 bar, que se utilizan para separar moléculas en un rango de peso molecular de 200 a $2000 \mathrm{~g} / \mathrm{mol}$ [65]. Las membranas de NF están diseñadas, generalmente, para ser selectivas por iones multivalentes en lugar de iones univalentes [5], además de esto se ha utilizado como una tecnología para el ablandamiento de agua y eliminación de metales con un tamaño de poro de $0.001 \mathrm{~mm}$, equivalente a $1 \mathrm{~nm}[12]$.

3.6.4. Ósmosis inversa (RO). Es un procedimiento impulsado por una presión diferencial mayor que la presión osmótica [66]. Las membranas de RO están diseñadas para rechazar todas las especies distintas del agua. Esta tecnología puede eliminar contaminantes más pequeños que el resto de membranas y se obtienen efluentes de alta calidad [12]; sin embargo, son incapaces de ofrecer una barrera significativa a los gases disueltos y a ciertas moléculas orgánicas de bajo peso molecular [5], el tamaño del poro se encuentra entre 5-15 A, permitiendo retener un contenido máximo de sólidos del 30 \% [64].

El tipo de membrana más común utilizado en los procesos de ósmosis inversa es la membrana semipermeable [66]. Los sistemas de membrana de ósmosis inversa tienen una vida útil entre tres y siete años. Sin embargo, las incrustaciones de membrana y el escalado son un punto crítico en este proceso, junto con el alto costo de requisitos de presión y capital [12]. 
En Colombia los tratamientos de agua de producción están enfocados, principalmente, a procesos convencionales de tipo físico y químico, como el desnatado, flotación por aire disuelto, filtración, oxidación química, entre otros mencionados anteriormente. Hasta ahora no se han desarrollado sistemas de tratamiento avanzados (no convencionales) que incluyan nuevas tecnologías o configuraciones integradas tales como los sistemas híbridos, los cuales comprenden dos procesos diferentes que, al integrarse en uno solo, permiten mejorar significativamente la eficiencia y operatividad del tratamiento de agua [67].

Como se mencionó previamente el reúso del agua de producción para aplicaciones agrícolas es una alternativa que puede traer beneficios tanto para los usuarios urbanos, agricultores como para el medioambiente, garantizando una gestión sostenible y eficiente de este recurso. Sin embargo, se debe tener en cuenta que para alcanzar este objetivo es necesario cumplir con los estándares de calidad y requerimientos estrictos dados por la normativa descrita en el siguiente apartado.

\subsection{Análisis Normativo para Aguas de Riego en Colombia} Para la reutilización de agua tratada en riego, es necesario considerar la normativa colombiana vigente, la cual presenta los límites y parámetros permisibles para la disposición final de este tipo de efluentes. Actualmente, la Resolución 1207 del 2014 establece las disposiciones relacionadas con el uso del agua residual tratada, esta presenta los límites permisibles para la reutilización de este recurso para usos agrícolas como se describen a continuación:

- Cultivos de pastos y forrajes para consumo animal.

- Cultivos no alimenticios para humanos o animales.

- Cultivos de fibras celulósicas y derivados.

- Cultivos para la obtención de biocombustibles (biodiesel y alcohol carburante), incluidos lubricantes.

- Cultivos forestales de madera, fibras y otros no comestibles.

- Cultivos alimenticios que no son de consumo directo para humanos o animales y que han sido sometidos a procesos físicos o químicos.

El agua residual tratada deberá cumplir previamente con los siguientes criterios de calidad mostrados en la tabla 1 :

Tabla 1. Valores máximos permisibles para disposición de agua residual en riego. Resolución 1207 del 2014

\begin{tabular}{|c|c|}
\hline Variable & $\begin{array}{c}\text { Valor máximo } \\
\text { permisible }\end{array}$ \\
\hline \multicolumn{2}{|l|}{ Físicos } \\
\hline $\mathrm{pH}$ & $6.0-9.0$ \\
\hline Conductividad ( $\mu \mathrm{S} / \mathrm{cm})$ & 1500 \\
\hline \multicolumn{2}{|l|}{ Microbiológicos } \\
\hline $\begin{array}{l}\text { Coliformes termotolerantes (NMP / } \\
100 \mathrm{~mL} \text { ) }\end{array}$ & $1 \times 10^{5}$ \\
\hline Enterococos fecales (NMP / 100 mL) & $1 \times 10^{2}$ \\
\hline $\begin{array}{l}\text { Helmintos parásitos humanos (hue- } \\
\text { vos y larvas / L) }\end{array}$ & 1.0 \\
\hline $\begin{array}{l}\text { Protozoos parásitos humanos (quis- } \\
\text { tes / L) }\end{array}$ & 1.0 \\
\hline Salmonella sp (NMP / 100 mL) & 1.0 \\
\hline \multicolumn{2}{|l|}{ Químicos } \\
\hline Fenoles totales (mg / L) & 1.5 \\
\hline Hidrocarburos totales (mg / L) & 1.0 \\
\hline \multicolumn{2}{|l|}{ Iones } \\
\hline Cianuro libre (mg CN- / L) & 0.2 \\
\hline Cloruro (mg Cl / L) & 300 \\
\hline Fluoruros (mg Fl- / L) & 1.0 \\
\hline Sulfatos ( $\mathrm{mg} \mathrm{SO}_{4}^{2-} / \mathrm{L}$ ) & 500 \\
\hline \multicolumn{2}{|l|}{ Metales } \\
\hline Aluminio (Al) (mg Al / L) & 5.0 \\
\hline Berilio (Be) (mg Be / L) & 0.1 \\
\hline Cadmio (Cd) (mg Cd / L) & 0.01 \\
\hline Cinc (Zn) (mg Zn / L) & 3.0 \\
\hline Cobalto (Co) (mg Co / L) & 0.05 \\
\hline Cobre (Cu) (mg Cu / L) & 1.0 \\
\hline $\operatorname{Cromo}(\mathrm{Cr})(\mathrm{mg} \mathrm{Cr} / \mathrm{L})$ & 0.1 \\
\hline Hierro (Fe) (mg Fe / L) & 5.0 \\
\hline
\end{tabular}




\begin{tabular}{|c|c|}
\hline Mercurio (Hg) (mg Hg / L) & 0.002 \\
\hline Litio (Li) (mg Li / L) & 2.5 \\
\hline Manganeso (Mn) (mg Mn / L) & 0.2 \\
\hline Molibdeno (Mo) (mg Mo / L) & 0.07 \\
\hline Níquel (Ni) (mg Ni / L) & 0.2 \\
\hline Plomo (Pb) (mg Pb / L) & 5.0 \\
\hline Sodio (Na) (mg Na / L) & 200 \\
\hline Vanadio (V) (mg V / L) & 0.1 \\
\hline \multicolumn{2}{|l|}{ Metaloides } \\
\hline Arsénico (As) (mg As / L) & 0.1 \\
\hline Boro (B) (mg B / L) & 0.4 \\
\hline \multicolumn{2}{|l|}{ No metales } \\
\hline Selenio (Se) (mg Se / L) & 0.02 \\
\hline \multicolumn{2}{|l|}{ Otros parámetros } \\
\hline $\begin{array}{l}\text { Cloro total residual (con mínimo } 30 \\
\text { minutos de contacto) }\left(\mathrm{mg} \mathrm{Cl}_{2} / \mathrm{L}\right)\end{array}$ & Menor a 1.0 \\
\hline Nitratos $\left(\mathrm{NO}_{3}-\mathrm{N}\right)(\mathrm{mg} / \mathrm{L})$ & 5.0 \\
\hline
\end{tabular}

\section{CONCLUSIONES}

El alto volumen de agua generado en la etapa de producción de la industria petrolera ha traído consigo estrategias para el manejo y gestión del recurso. Actualmente, en Colombia las técnicas más utilizadas para disposición final de este efluente son la inyección y el vertimiento superficial; sin embargo, se han desarrollado algunos proyectos para generar un valor agregado a este recurso, como el reúso en actividad agrícola. Para este fin se requieren sistemas de tratamiento robustos que cuenten con operaciones de pulido, permitiendo al agua alcanzar los requerimientos de calidad exigidos en la normativa.

La incorporación de tratamientos no convencionales como tecnologías de membrana (ósmosis inversa), procesos de oxidación avanzada (fotofenton), procesos de biorremediación (humedales artificiales), electrodiálisis y adsorción (carbón activado) a los sistemas de tratamiento actuales son indispensables para obtener un efluente de calidad que pueda disponerse en riego. Es importante continuar desarrollando proyectos de investigación en técnicas no convencionales e implementando diferentes tecnologías de tratamiento a escala piloto, con el fin de avanzar en procesos de desarrollo sostenible y economía circular.

\section{RECOMENDACIONES}

Evaluar los requerimientos posteriores al sistema de tratamiento para la disposición del agua de producción tratada en riego a cultivos agroenergéticos.

\section{REFERENCIAS}

[1] J. O. Robertson, and G. V. Chilingar, Environmental aspects of oil and gas production. John Wiley \& Sons, 2017.

[2] Water Environment Federation. "Oil and gas terminology glossary". Monthly Energy Review, 91, 2018.

[3] J. García, M. Cabarcas, y S. Herrera. "Manejo del agua de producción para proyectos de gas en aguas profundas y ultraprofundas del caribe colombiano". El Reventón Energético, 15(2), 89-105, 2017. https://revistas.uis.edu.co/index.php/revistafuentes/article/view/7686/8698 DOI: https://doi. org/10.18273/revfue.v15n2-2017008

[4] L. Nabzar, and J. Duplan. "Water in fuel production: Oil production and refining". Panorama 2011, IFP Energies, 2011.

[5] A. Fakhru'l-Razi, A. Pendashteh, L. C. Abdullah, D. R. A. Biak, S. S. Madaeni, and Z. Z. Abidin. "Review of technologies for oil and gas produced water treatment". Journal of Hazardous Materials, 170(2-3), 530-551, 2009. DOI: https://doi.org/10.1016/j.jhazmat.2009.05.044

[6] E. F. Almansa-Manrique, J. G. Velásquez-Penagos, y G. A. Rodríguez-Yzquierdo. "Efecto del uso de aguas provenientes de la producción petrolera en actividades agrícolas y pecuarias". Corpoica Ciencia y Tecnología Agropecuaria, 19(2), 403-420, 2018. DOl: https://doi.org/10.21930/rcta.vol19_num2_art:1016

[7] Ecopetrol. Reporte Integrado de Gestión Sostenible 2019. 
http://dev.isgood.com.co/ecopetrol/informe-2019/ es/

[8] BNamericas. "Colombia's 20 highest producing oil fields". BNamericas, 2019. https://www.bnamericas.com/en/news/colombias-20-highest-producing-oilfields.

[9] C. Gil y B. Mendoza. "Diseño de un modelo de ingeniería para la gestión del agua excedente del campo Castilla a fin de disponerla en cultivos que generan biocombustibles, según la Resolución 1207 de 2014", Trabajo de grado, Universidad de América, 2019.

[10] E. Igunnu, and G. Z. Chen. "Produced water treatment technologies". International Journal of Low-Carbon Technologies, 9(3), 157-177, 2012. DOI: https://doi.org/10.1093/ijlct/cts049

[11] T. Hayes, and D. Arthur. Overview of emerging produced water treatment technologies. The 11th Annual International Petroleum Environmental Conference, Albuquerque, NM, October 12-15, 2004. https:// www.yumpu.com/en/document/read/33648850/ overview-of-emerging-produced-water-treatment-technologies-ipec

[12] S. Jiménez, M. Micó, F. Medina, M. Arnaldos, and S. Contreras. "State of art of produced water treatment". Chemosphere, 192, 186-208, 2018. https://www.sciencedirect.com/science/article/ abs/pii/S0045653517317241 DOI: https://doi.org/10.1016/j.chemosphere.2017.10.139

[13] J. Neff. "Produced water". In Bioaccumulation in marine organisms: Effect of contaminants from oil well produced water. Elsevier, 1-35, 2002. DOI: https:// doi.org/10.1016/B978-0-08-043716-3.X5000-3

[14] M. Stewart, and K. Arnold. Part 1 - "Produced water treating systems". InProduced waterfield manual. Gulf Professional Publishing, 1-134, 2011. DOI: https:// doi.org/10.1016/B978-1-85617-984-3.00001-8

[15] S. L. Mesa, J. M. Orjuela, A. T. Ortega Ramírez y J. Sandoval. "Revisión del panorama actual del manejo de agua de producción en la industria petrolera colombiana". Gestión y Ambiente, 21(1), 87-98. 2018. DOI:https://doi.org/10.15446/ga.v21n1.69792

[16] A. Ortega. "Estrategia para el uso sostenible de agua de producción para riego de suelos, adaptada de la experiencia del desierto de Omán a un patrón de pozos en un campo colombiano". Tesis de Maestría. Universidad de América, 2019.

[16] A. Ortega. "Estrategia para el uso sostenible de agua de producción para riego de suelos, adaptada de la experiencia del desierto de Omán a un patrón de pozos en un campo colombiano". Tesis de Maestría. Universidad de América, 2019.

[17] N. Lusinier, I. Seyssiecq, C. Sambusiti, M. Jacob, N. Lesage, and N. Roche. "Biological treatments of oilfield produced water: A comprehensive review". Society of Petroleum Engineers Journal, 24(5), 21352147, 2019. DOI: 10.2118/195677-PA

[18] M. Srijata, and R. Pranab. "BTEX: A serious ground-water contaminant". Research Journal of Environmental Sciences, 5(5), 394-398, 2011. DOI: 10.3923/rjes.2011.394.398

[19] Asociación Regional de Empresas de Petróleo y Gas Natural en Latinoamérica y el Caribe ACIPET. "Disposición y tratamiento del agua producida". Guía Ambiental No. 1, 2006. http://www.ingenieroambiental.com/4000/GUIA\%2001\%20-\%200K.pdf

[20] D. A. Puentes Álvarez y J. S. Velasco García. "Evaluación de la viabilidad de aplicación de la tecnología radio corto para la perforación de un pozo en el campo Rubiales". Trabajo de grado. Universidad de América, 2018.

[21] Schlumberger. "FWKO". Oilfield Glossary, 2020. https://www.glossary.oilfield.slb.com/es/terms/f/ fwko

[22] P. Grill, and F. Linde. "Oil skimming: Business potential, and strategic options facing a marginalized business segment at Sandvik Process Systems". Master of Science Thesis, KTH School of Industrial Engineering and Management, 2013. Disponible en: http:// www.diva-portal.org/smash/get/diva2:644788/FULLTEXT01

[23] M. V. Madriz. "Estudio técnico, económico y financiero de la implementación de plantas modularizadas de tratamiento de aguas aceitosas como producto de la extracción de petróleo para una 
empresa de proyectos de Ingeniería Procura y Construcción (IPC)". Trabajo de grado. Universidad Católica Andrés Bello, 2014. https://1library.co/document/y654407z-aas.html

J. Forero, J. Diaz, y V. Blandón. "Diseño de un nue-

[24] vo sistema de flotación para tratamiento de aguas industriales". CTF - Ciencia, Tecnología y Futuro, 1(5), 67-75, 1999. Disponible en: http://www. scielo.org.co/scielo.php?script=sci_arttext\&pi$d=S 0122-53831999000100006 \& \mid n g=e n \& t \operatorname{lng}=e s$

[25] D. García y P. Vivas. (2016). “Ampliación de la capacidad del sistema de tratamiento de agua de producción mediante el dimensionamiento conceptual de los nuevos equipos en la estación Jaguar ubicada en el bloque Caracara". Trabajo de grado. Universidad de América, 2016.

[26] J. Cantos. "Diseño de una planta para el tratamiento de agua de formación para su reinyección en el campo Sacha". Trabajo de grado. Escuela Politécnica Nacional, 2017.

[27] Ecopetrol. Filosofía de operación filtros de cáscara de nuez estación Apiay y Suria, 2010.

[28] E. Piñeiro, R. Fonseca y J. Ruiz. “Diseño, construcción e instalación de un reactor para depurar aceites vegetales usados para ser reutilizados en calderas y hornos". Trabajo de grado. Universidad Nacional de la Amazonía Peruana, 2013.

[29] D. Hincapié y S. Verján. "Evaluación técnica de la inyección cíclica de agua en la formación K1 inferior del campo Castilla mediante simulación numérica". Trabajo de grado. Universidad de América, 2019.

[30] C. Quintero Gómez. "Alternativas de producción más limpia en la estación de recolección y tratamiento Castilla II Superintendencia de Operaciones Apiay - Soa. Departamento del Meta. Ecopetrol S. A.". Trabajo de grado. Universidad de la Salle, 2007.

[31] J. Torres y D. Tosi. "Diseño y elaboración de un prototipo para trampa de grasa, aplicable al sector comercial de la ciudad de Cuenca". Trabajo de grado. Universidad del Azuay, 2019.

[32] R. Mastouri, S. M. Borghei, F. Nadim, and E. Roayaei. "The investigation of induced gas flotation (IGF) performance at elevated temperatures and high total dissolved solids (TDS) produced water". Petroleum Science and Technology, 28, 1415-1426, 2009. Disponible en: https://www.researchgate. net/publication/228504759_THE_INVESTIGATION_ OF_INDUCED_GAS_FLOTATION_IGF_PERFORMANCE_AT_ELEVATED_TEMPERATURES_AND_HIGH_ TOTAL_DISSOLVED_SOLIDS_TDS

[33] Process Group. "Induced Gas Flotation D.04", 2012. Disponible en: http://www.processgroupintl.com/ media/downloads/D04_Gas_Flotation_rev_11-12. pdf

[34] R. Hoseinzadeh, A. Hossein, M. Omidkhah, and M Sharifzadeh. "An economical comparative study of different methods for decrease towers makeup cost in oil refineries". World Applied Sciences Journal, 12(7), 2011. Disponible en: https://www. researchgate.net/publication/268380429_An_ Economical_Comparative_Study_of_Different_Methods_for_Decrease_Cooling_Towers_Makeup_ Cost_in_Oil_Refineries

[35] J. Winpenny, I. Heinz y S. Koo-Oshima. "Reutilización del agua en la agricultura: ¿Beneficios para todos?" Informe sobre Temas Hídricos FAO 35, 2013. Disponible en: http://www.fao.org/3/a-i1629s.pdf

[36] R. Arnold, D. Burnett, J. Elphick, T. Feely, M. Galbrun, M. Hightower, Z. Jiang, M. Khan, M. Lavery, F. Luffey y P. Verbeek. "Manejo de la producción de agua: De residuo a recurso". Oilfield Review, 2004. Disponible en: https://www.academia.edu/33018242/Manejo_de_la_producci\%C3\%B3n_de_agua_De_residuo_a_recurso

[37] E. Salamanca. "Tratamiento de aguas para el consumo humano". Módulo Arquitectura CUC, 17(1), 29-48, 2016. DOI: https://doi.org/10.17981/moducuc.17.1.2016.02

[38] E. Worch. "Adsorption technology in water treatment: Fundamentals, processes and modeling." Walter de Gruyter, 2012. DOI: ttps://doi. org/10.1515/9783110240238

[39] M. A. Al-Ghouti, M. A. Al-Kaabi, M. Y. Ashfaq, and D. A. Da'na. "Produced water characteristics, treatment 
and reuse: A review". Journal of Water Process Engineering, 28, 222-239, 2019. DOI: https://doi.org/10.1016/j.jwpe.2019.02.001

[40] C. Rawlins, "Experimental study on oil and solids removal in nutshell filters for produced water treatment". In SPE Western Regional Meeting, Garden Grove, California, USA, April 2018. DOI: 10.2118/190108-ms

[41] M. Nasiri, and I. Jafari. "Produced water from oil-gas plants: A short review on challenges and opportunities". Periodica Polytechnica Chemical Engineering, 61(2), 2017. DOI: 10.3311/ppch.8786

[42] A. Heydari, R. Duraisamy, and A. Henni. "State of the art treatment of produced water". Water Treatment. 2013. DOI: 10.5772/53478

[43] T. Pankratz. "Evaporation: A wastewater treatment alternative: Wastewater Treatment". Roads \& Brigdes, December 28, 2000. DIsponible en: https:// www.roadsbridges.com/evaporation-wastewater-treatment-alt

[44] A. Azevedo, H. A. Oliveira, and J. Rubio. "Treatment and water reuse of lead-zinc sulphide ore mill wastewaters by high-rate dissolved air flotation". Minerals Engineering, 127, 114-121, 2018. DOI: 10.1016/j.mineng.2018.07.011

[45] A. Al-Shamrani, A. James, and H. Xiao. "Separation of oil from water by dissolved air flotation". Colloids and Surfaces A: Physicochemical and Engineering Aspects, 209(1), 15-26, 2002. doi:10.1016/s09277757(02)00208-x

[46] M. Nasiri, I. Jafari, and B. Parniankhoy. "Oil and gas produced water management: A review of treatment technologies, challenges, and opportunities". Chemical Engineering Communications, 204(8), 9901005, 2017. DOI: 10.1080/00986445.2017.1330747

[47] T. Xu, and C. Huang. "Electrodialysis-based separation technologies: A critical review". AlChE Journal, 54(12), 3147-3159, 2008. DOI: 10.1002/aic.11643

[48] L. K. Wang, D. Vaccari, Y. Li y N. K. Shammas. "Chemical precipitation". In: L. K. Wang, Y. T. Hung, and N. K. Shammas (eds.) Physicochemical treatment processes. Handbook of Environmental Engineering, vol 3. Humana Press, 2005. DOI: https://doi.org/10.1385/1-59259-820-x:141

[49] M. S. Oncel, A. Muhcu, E. Demirbas, and M. Kobya. "A comparative study of chemical precipitation and electrocoagulation for treatment of coal acid drainage wastewater". Journal of Environmental Chemical Engineering, 1(4), 989-995, 2013. DOI: 10.1016/j.jece.2013.08.008

[50] T. Muddemann, D. Haupt, M. Sievers, and U. Kunz. "Electrochemical reactors for wastewater treatment". ChemBioEng Reviews, 6(5), 142-156, 2019 DOI: 10.1002/cben.201900021

[51] E. Gilpavas, E. Arbeláez, L. Sierra, C. White, C. Oviedo y P. Restrepo. "Aplicación de la electroquímica en el tratamiento de aguas residuales". Cuadernos de Investigación. Universidad EAFIT, 2008.

[52] A. M. Al-Sabagh, N. Kandile, and M. R. Noor El-Din. "Functions of demulsifiers in the petroleum industry". Separation Science and Technology, 46(7), 11441163, 2011. DOI: :10.1080/01496395.2010.550595

[53] H. N. Abdurahman, M. Y. Rosli, and J. Zulkifly. "Chemical demulsification of water-in-crude oil emulsions". Journal of Applied Sciences, 7: 196-201, 2007. DOI: 10.3923/jas.2007.196.201

[54] T. Luo, S. Abdu, and M. Wessling. "Selectivity of ion exchange membranes: A review". Journal of Membrane Science, 555, 429-454, 2018. DOI: 10.1016/j. memsci.2018.03.051

[55] S. Renou, J. G. Givaudan, S. Poulain, F. Dirassouyan, and P. Moulin. "Landfill leachate treatment: Review and opportunity". Journal of Hazardous Materials, 150(3), 468-493, 2008. DOI: 10.1016/j. jhazmat.2007.09.077 9

[56] N. K. Shammas, J. Y. Yang, P. C. Yuan, and Y. T. Hung. "Chemical oxidation". Physicochemical Treatment Processes, 229-270, 2005. DOI: 10.1385/1-59259820-x:229

[57] S. Shokrollahzadeh, F. Golmohammad, N. Naseri, H. Shokouhi, and M. Arman-Mehr. "Chemical oxidation for removal of hydrocarbons from gas-field produced water". Procedia Engineering, 42, 942-947, 2012. DOI: https://doi.org/10.1016/j. proeng.2012.07.487 
[58] United States Environmental Protection Agency EPA. "Ozone disinfection" Wastewater Technology Fact Sheet, 1999. Disponible en: https://www3.epa. gov/npdes/pubs/ozon.pdf

[59] Y. Gong, and D. Zhao. "Effects of oil dispersant on ozone oxidation of phenanthrene and pyrene in marine water". Chemosphere, 172, 468-475, 2017. DOI: 10.1016/j.chemosphere.2017.01.007

[60] A. B. C. Alvares, C. Diaper, and S. A. Parsons. "Partial oxidation by ozone to remove recalcitrance from wastewaters: A review". Environmental Technology, 22(4), 409-427, 2001. DOI: 10.1080/09593332208618273

[61] Y. Deng, and R. Zhao. "Advanced oxidation processes (AOPs) in wastewater treatment". Current PoIlution Reports, 1(3), 167-176, 2015. DOI: 10.1007/ s40726-015-0015-z

[62] Y. Barash. "Biological treatment of produced water". AWE Magazine, October 3, 2010. Disponible en: https://www.aweimagazine.com/article/biological-treatment-of-produced-water-237/

[63] H. T. Madsen. "Membrane filtration in water treatment: Removal of micropollutants". In Chemistry of advanced environmental purification processes of water, Elsevier, 199-248, 2014. DOI: 10.1016/b9780-444-53178-0.00006-7

[64] C. A. Solís, C. A. Vélez y J. S. Ramírez-Navas. "Tecnología de membranas: Ultrafiltración". Entre Ciencia e Ingeniería, 11(22), 26-36, 2017. DOI: https://doi. org/10.31908/19098367.3546

[65] C. Charcosset. "Some perspectives". Membrane Processes in Biotechnology and Pharmaceutics, 295-321, 2012. DOI: 10.1016/b978-0-444-563347.00008-3

[66] Y. Mikhak, M. M. A. Torabi, and A. Fouladitajar. "Refinery and petrochemical wastewater treatment". Sustainable Water and Wastewater Processing, 55-91, 2019. DOI: 10.1016/b978-0-12-8161708.00003-x

[67] E. Vicuña, S. Ara y J. Loayza. "Sistemas híbridos de tratamiento de aguas residuales". Revista Peruana de Química e Ingeniería Química, 